\title{
Link Adaptation Algorithm for IEEE 802.11 Wireless Local Area Netwoks in Fading Channel
}

\author{
Wan Norsyafizan W Muhamad, Suzi Seroja Sarnin, Azlina Idris, Aliya Syahira \\ Universiti Teknologi MARA, 40450 Shah Alam, Selangor, Malaysia
}

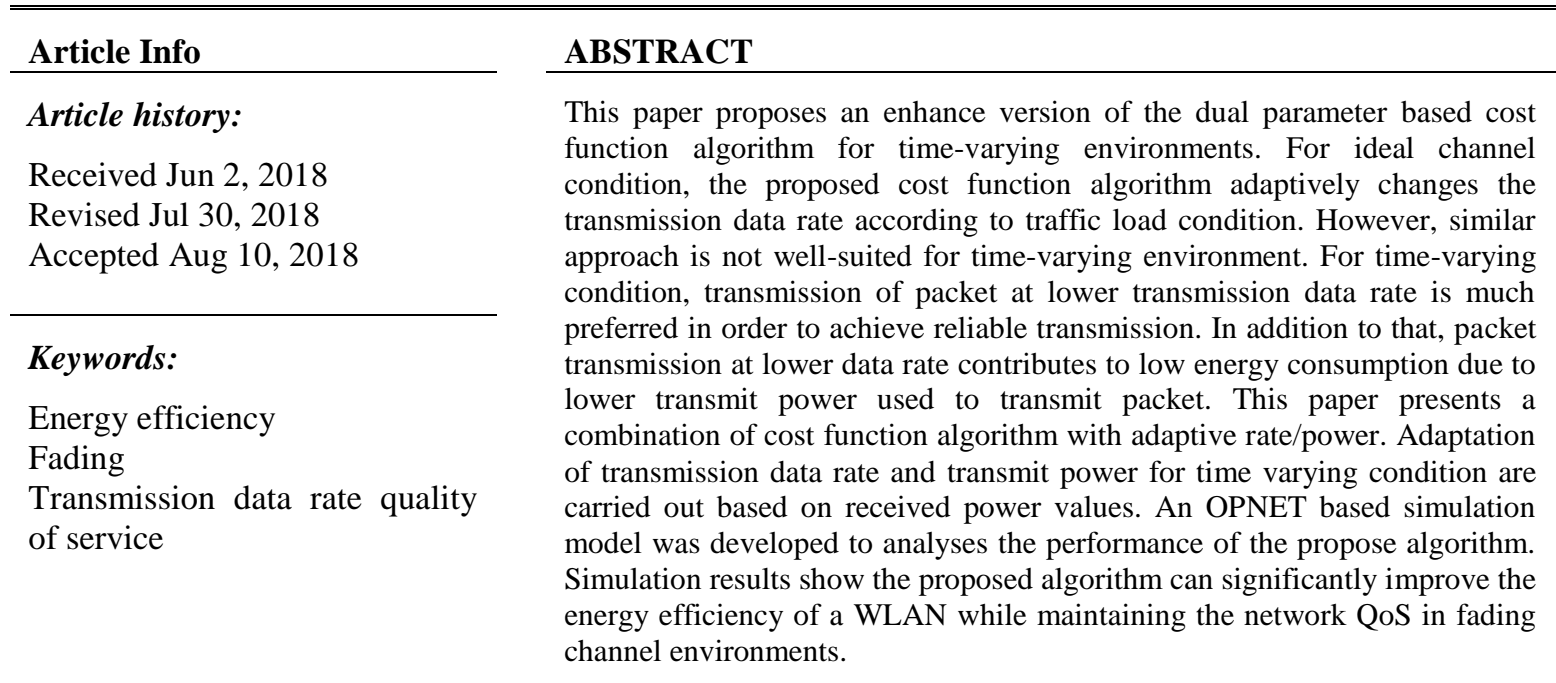

Copyright $(0) 2018$ Institute of Advanced Engineering and Science. All rights reserved.

\section{Corresponding Author:}

Wan Norsyafizan W Muhamad

Universiti Teknologi MARA,

40450 Shah Alam, Selangor, Malaysia.

Email: syafizan@salam.uitm.edu.my

\section{INTRODUCTION}

In recent years, the conservation of energy has become one of the critical current research areas due to increases in the deployment of network infrastructure in parallel with the rising demands of Information and Communication Technology (ICT) services. Based on recent surveys in [1-2], from 2012 to 2017, it is forecast that global average internet traffic growing at an annual rate of $23 \%$. Increased energy consumption in wireless networks contributes to increased greenhouse gas emissions and higher operating cost. According to energy efficiency studies [3], the carbon footprint is estimated around 1\%-2\% globally. In addition to a rise in greenhouse gases, business operation costs could also increase. Hence, it is important to develop energy efficient techniques for future communication networks.

A considerable amount of energy saving techniques for the IEEE802.11 based WLAN networks have been presented in the state of the art literature. Energy efficiency can be improved by incorporating advanced algorithm in the different layers of the protocol stack such as the transmission power control (TPC) [4] and physical layer rate adaptation techniques [5-6]. The link adaptation mechanism offers the ability to adapt the modulation and coding scheme (MCS) based on channel conditions on the radio link or also called as channel state information (CSI) [7-8]. Traditionally in WLANs, the technique is used to maximize the throughput by transmitting at the maximum possible transmission data rate [9]. However, in fading channel, lower data rates are preferred to combat with higher interference [10]. An accurate indicator of the channel quality is an important parameter for the rate adaptation. Some of the proposed techniques used signal to noise ratio as an indicator for the rate adaptation [11]. In time-varying channel conditions, there are also 
techniques proposed in literature which adapts the data rate based on received signal strength indicator [12]. In the literature, various techniques have been used to quantify the fading conditions such as presented in [6, 13, 14, 15]. Raja et al [6] propose an adaptive learning mechanism, HT-MobiRate, for high throughput dynamic link adaptation under mobile scenario. As the most widely used standards for wireless local area network (WLAN), IEEE 802.11 standards are continuously amended by introducing new techniques as to meet the increasing demands $[13,16]$. To verify the performance of amended protocols, network simulation is considered as a significant method. Wensheng et al [17] design and further implement the integrated linksystem level simulation platform, which makes it possible to evaluate the new technologies for IEEE 802.11ax.

This paper proposes the link adaptation based dual parameter cost function algorithm to deal with fading environments. The paper aims to apply the previously developed cost function algorithm with additional features to combat with the varying channel conditions. The main objective of enhance dual cost function algorithm for fading channel is to improve the energy efficiency while maintaining the QoS performances. These is achieved by limiting the data rate and transmit power which results from the cost function algorithm to an appropriate level to ensure successful packet transmission at the receiver site. Thus, in fading condition results of transmission data rate from the cost function algorithm (in ideal channel condition) is again adapted based on channel environment (represents by the average received power) and new data rate will vary from the minimum level up to the ceiling rate only.

\section{RESEARCH METHOD}

This section describes in details enhance dual cost function algorithm with additional features to overcome the fading environments. The enhance technique apply the previously developed cost function algorithm (as presented for ideal channel condition) [14] with additional features of adaptive rate/power according to the channel condition which represents by the value of received signal strength.

\subsection{Dual Parameter Cost Function Algorithm}

The dual parameter cost function algorithm as proposed in [14], initially was developed for ideal channel conditions. In ideal channel condition, the decision of the rate/power selection is solely depends on the value of the cost function, $F(t, Q)$. The cost function value $F(t, Q)$ incorporates the values of measured delay, current queue, queue threshold and a tunable parameter, which is the rate adaptation sensitivity parameter. The function $\mathrm{F}(\mathrm{t}, \mathrm{Q})$ is represented as:

$$
\begin{aligned}
& F(t, Q) \\
& = \begin{cases}C\left(\frac{1}{T_{\text {target }}-T_{\text {current }}} \times\left(\frac{Q_{\text {current }}, j}{Q_{\text {threshold }, j}}\right)\right) & \text { for } T_{\text {crt }}<T_{\text {target }} \\
1 & \text { for } T_{\text {crt }} \geq T_{\text {target }, j}\end{cases}
\end{aligned}
$$

The novelty of the cost function algorithm is a rate selection based on dual parameter approach, where a cost function takes account an average measured delay of the traffic and the queue length of an individual transmitter. From the equation, $\mathrm{C}$ is a rate adaptation sensitivity factor, Ttarget is the target delay, Tcurrent is the measured delay in the network. Qcurrent, $\mathrm{j}$ is the jth terminal transmission queue length. The queue threshold Tthreshold is measured as a short-term average queue length calculated in the past averaging period. The short-term average queue size is updated for every $\mathrm{N}$ packets, based on Equation (2). Qi is an instantaneous queue length for packet $i$.

$$
Q_{\text {threshold }}=\frac{1}{(N-1)} \sum_{i=1}^{N-1} Q_{i}
$$

The cost function algorithm adjusts the transmission data rate according to dual transmission parameters, which are the end to end delay and the queue length. This is achieved by mapping the function of $\mathrm{F}(\mathrm{t}, \mathrm{Q})$ based on the data rate mapping table, as in Table 1 [14]. At very light loads, the function of $F(t, Q)$ results in lower values, thus adapting to a range of lower data rates. As the traffic increases, function $F(t, Q)$ shows significant rises, thereby adapting to higher levels of the transmission data rates. Following that, an 
appropriate transmission power which corresponds to the selected data rate is applied. However, the same concept of adapting the transmission data rate in ideal channel conditions could not always be performed in a non-zero BER channel due to rapid variations of the received signal power. In the case of destructive fading, the signal experienced a significant drop thus the combination of the cost function algorithm and an adaptive rate/power technique based on received signal value needs to be utilized according to the channel condition.

\subsection{Enhance Features: Adaptive Rate/Power for Fading Channel}

In order to combat with varying channel condition in real environments, the new rate adaptation known as adaptive rate/power in fading channel is performed using a threshold based scheme which switches between multiple transmission data rates depending on the received signal value. The threshold for the rate selection is based on the minimum required power level for each of the MCS values, which ensures the correct reception of a packet at the receiver. The threshold is set according to that value because it is the minimum received power value to decode the packet correctly at the receiver for each specific data rate. If the received power is below the required level, the packet cannot be received successfully at that transmission data rate. Based on the rate adaptation (threshold scheme), the current transmission data rate that has been selected according to cost function algorithm will be limited by the appropriate data rate Rmax, based on the received power values. Details of the proposed adaptive rate adaptation for fading conditions are demonstrated in pseudo code:

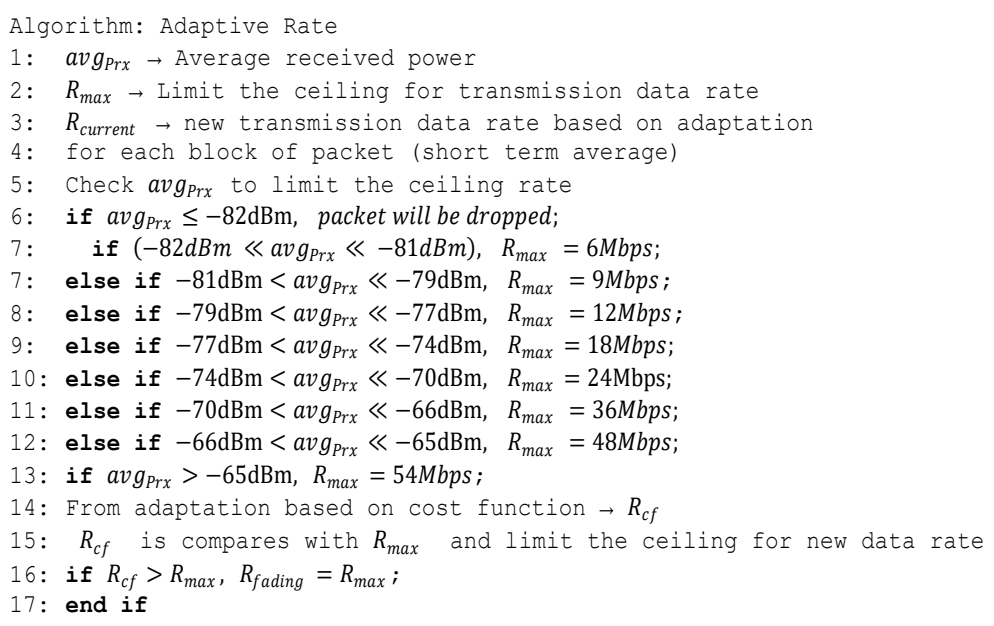

The power adaptation is performed based on the received signal value. Due to the effects of multipath fading, the adaptation of the transmit power can either increase or decrease the existing transmit power with a specific margin based on the fluctuations of the received signal power, as in Equation (3):

$$
\text { Ptx } x_{\text {fading }}=\text { Ptx } x_{\text {current }} \pm \Delta \text { margin }
$$

The $\Delta$ margin can have positive or negative values, depending on either constructive or destructive fading is occurring. If destructive fading occurs, then the $\Delta$ margin will be a positive value, which indicates that the initial power for the fading condition needs to be increased to overcome the effects of the fading. This is done based on the received power value. Take for example, if the current transmission data rate from the cost function algorithm is $\mathrm{R}$, then the minimum received power to decode the packet correctly at that data rate is known, assume that Prxmin. Based on the channel estimation, the average received power value Prxavg is compared with the minimum received power at the specific data rate. If the current average received power value from the channel estimation is less than the minimum received power at the specific data rate (Prxavg <= Prxmin), this indicates that the channel is deteriorated, hence the initial power will be increased by one level above the current power level, based on the lookup table. The lookup table is calculated offline which is the minimum transmit power required for each of the MCS value. In contrast, if constructive fading occurs, where the average received power is more than the minimum received power at that data rate (Prxavg > Prxmin), which specifies a good channel condition, then the transmit power either remains at the existing power level or decreases one step in order to gain an advantage of lower energy consumption. To give better understanding of how the power level is selected, theoretically the lookup table is created where the transmit power value for each of the MCS is selected. For this lookup table, the received power values for each of the MCS is assumed as the minimum received power to ensure correct reception at 
specific data rate and the transmission distance is assumed to be $200 \mathrm{~m}$ for path loss calculation. Table 1 summarized values of transmit power selected for each of transmission data rate for both case which are ideal and fading channel conditions.

Table 1. Lookup table for transmit power values

\begin{tabular}{|c|c|c|}
\hline $\begin{array}{c}\text { Transmission Data Rate } \\
(\mathrm{Mbps})\end{array}$ & $\begin{array}{c}\text { Transmit Power (Watt) Ideal } \\
\text { channel condition }\end{array}$ & $\begin{array}{c}\text { Transmit Power (Watt) Fading } \\
\text { channel condition }\end{array}$ \\
\hline 6 & 0.002 & 0.004 \\
\hline 9 & 0.003 & 0.005 \\
\hline 12 & 0.005 & 0.007 \\
\hline 18 & 0.008 & 0.012 \\
\hline 24 & 0.016 & 0.025 \\
\hline 36 & 0.040 & 0.063 \\
\hline 48 & 0.102 & 0.158 \\
\hline 54 & 0.128 & 0.199 \\
\hline
\end{tabular}

\section{RESULTS AND ANALYSIS}

Performances of the proposed technique are evaluated using an OPNET based simulation model. The network model consists of ten user terminals (UE) and one Access Point (AP) placed randomly in a $200 \mathrm{~m} \times 200 \mathrm{~m}$ area. This section compares performances of the enhance cost function with adaptive rate/power in fading condition with previous cost function algorithm in ideal channel condition. For this algorithm, fading channel model is simulated with two different coefficient factors which are $(\mathrm{k}=1,3)$. Simulation of the proposed algorithms is performed with varying load conditions from $(p=0.1$ to $p=0.4)$. The parameters used in simulations are summarized in Table 2. Simulation results are averaged over 3 simulation runs.

Table 2. Key simulation parameters

\begin{tabular}{ll}
\hline Parameter & Value \\
\hline WLAN Standard & IEEE $802.11 \mathrm{~g}$ \\
Operating Frequency & $2.4 \mathrm{GHz}$ \\
Number of UEs & 10 \\
Transmission Range & $100 \mathrm{~m}-300 \mathrm{~m}$ \\
& Free space path loss \\
Propagation Model & Rician Fading model \\
& (coefficient factor, k=1,3) \\
QoS delay threshold & $200 \mathrm{~ms}$ \\
Packet delay threshold & $1-3 \%$ \\
Packet Generation & Exponentially distributed \\
Noise density & $-163 \mathrm{dBm} / \mathrm{Hz}$ \\
Packet length & $1000 \mathrm{bytes}$ \\
Transmit power (Ptx) & $745.2 \mathrm{~mW}$ \\
Receive power $($ Prx $)$ & $370.8 \mathrm{~mW}$ \\
Idle power $($ Pidle $)$ & $18 \mu \mathrm{W}$ \\
\hline
\end{tabular}

Figure 1 represent the received power values (in $\mathrm{dBm}$ ) with simulation time for both ideal and fading cases at $p=0.1$. It can be observed from the figure that the fading condition results in much lower received power values as compared to the ideal channel. For ideal channel condition, the overall received signal strength is above $-65 \mathrm{dBm}$, therefore all packets are able to be transmitted at all levels of transmission data rate thus contributing to $100 \%$ of packet success rate. In the case of fading condition, overall data rate will be at much lower level according to the received power values to ensure correct reception at the receiver site.

From simulation results, it is also can be observed that variation of received power for all cases are more than $-82 \mathrm{dBm}$ which reveals that there is no packet loss due to poor channel condition at these load as can be seen from Table 3. The table shows a comparison of the packet loss rate with varying loads for all scenarios. It can be seen from the table that the combined cost function shows higher packet losses compared to the ideal channel condition. 


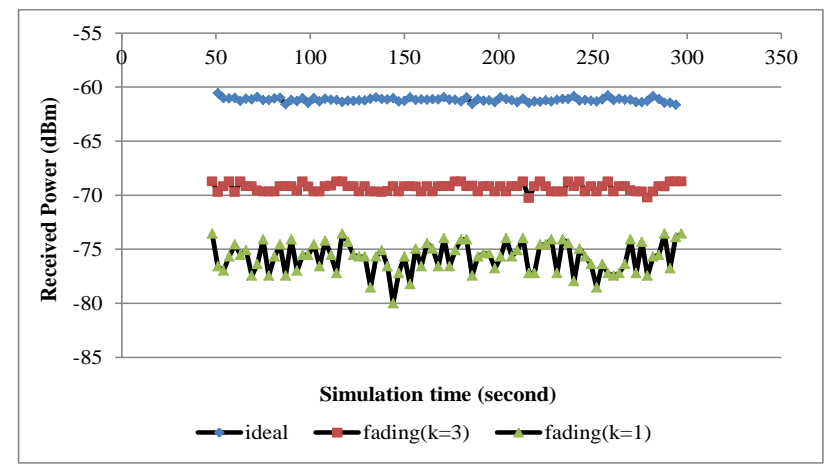

Figure 1. Variation of received power for ideal and fading channel at $p=0.1$

Table 3. Percentage of packet loss

\begin{tabular}{|c|c|c|c|}
\hline \multirow{2}{*}{ Network Load } & \multicolumn{3}{|c|}{ Packet Loss (\%) } \\
\cline { 2 - 4 } & $\mathrm{CF}($ ideal) & fading(k=3) & fading(k=1) \\
\hline 0.05 & 0 & 0 & 0 \\
\hline 0.1 & 0 & 0 & 0 \\
\hline 0.2 & 0 & 0 & 0 \\
\hline 0.25 & 0 & 0 & 0 \\
\hline 0.3 & 0.23 & 0.8 & 1.5 \\
\hline 0.4 & 0.3 & 1.3 & 2.3 \\
\hline
\end{tabular}

Figure 2 plots the variation of received power values for fading channel with $(k=3)$. For fading condition, the proposed combination of cost function and adaptive rate/power limits the data rate according to the fluctuation of received power values as plots in Figure 3. Based on the proposed algorithm as explained, as long as the data rate which result from the cost function algorithm does not reach the maximum level as in Figure 3, the data rate can stay at the same level. If it is more than the ceiling rate, then it will stay at maximum level which is either $24 \mathrm{Mbps}$ or $36 \mathrm{Mbps}$ in this case as can be seen from Figure 4. After selection of more robust rate according to the fluctuation of received power values then the transmit power is reduced to the level appropriate for that specific MCS value which ensure correct reception as shown in Figure 5.

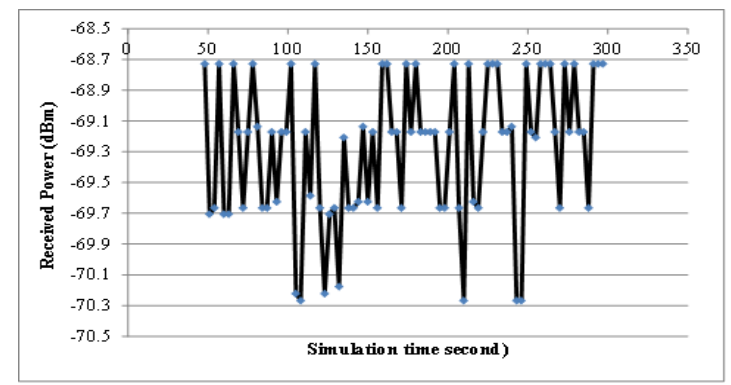

Figure 2. Variation of received power value for fading condition $(\mathrm{k}=3)$

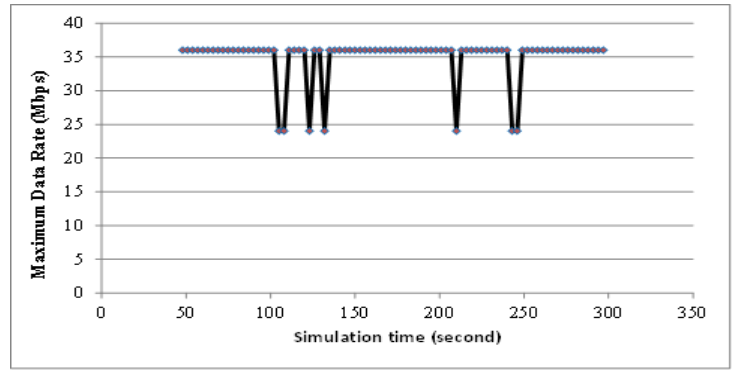

Figure 3. Transmission data rate maximum based on received power values 


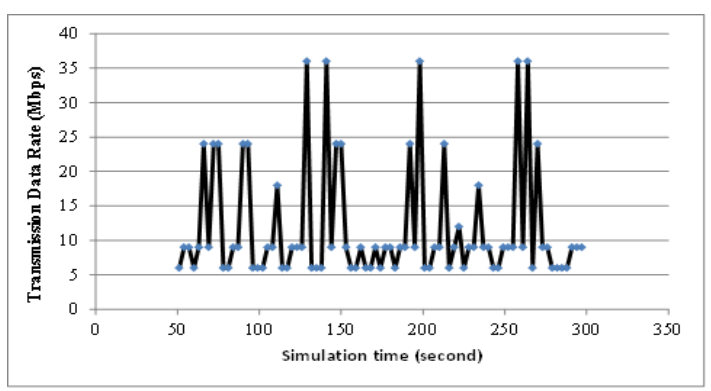

Figure 4. Adaptation of transmission data rate for fading condition

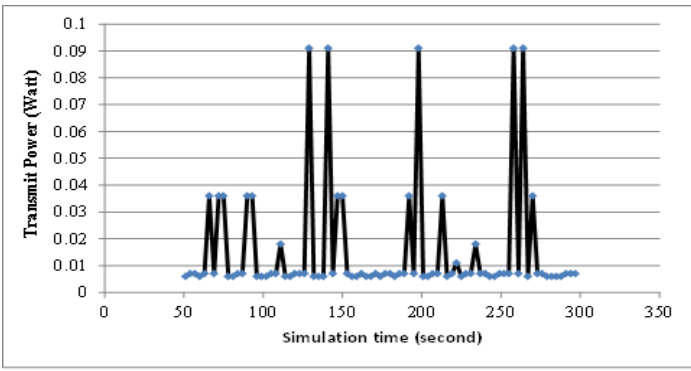

Figure 5. Adaptation of transmit power for fading condition

Figure 6 show the distribution of transmission data rates for both the adaptation cases at a specific load, $p=0.1$. It can be observed that the adaptation fading channel shows higher percentages at lower data rates than the adaptation cost function for the ideal channel. This result can be correlated to the results for variations of the received signal strength with corresponding transmission data rates for the fading channel, as shown in Figure 2.

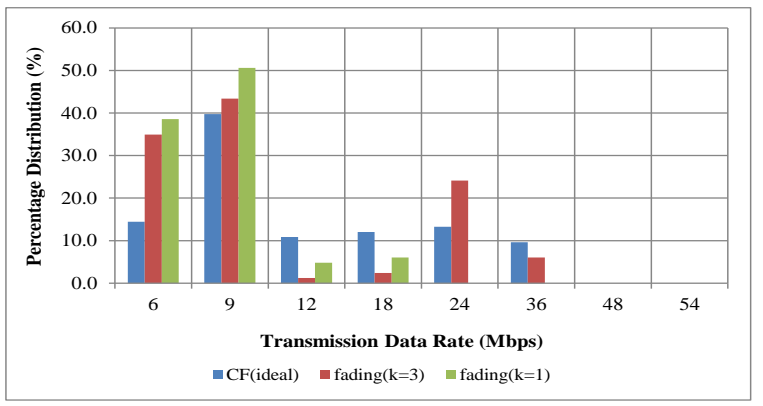

Figure 6. Distribution data rates for both adaptation cases at $\mathrm{p}=0.1$

Figure 7 plots the energy per bits for all conditions. From the figure it can be seen that energy saving for fading condition is achieved at higher load conditions. This is because at low load example at $(p=0.05$ and $p=0.1$ ), both techniques results in almost same data rate values. At low load, when contention is not an issue yet, lower value of queue and delay leads to lower $F(t, Q)$ value thus adapting to lower data rate for the cost function algorithm. In fading condition, after the algorithm compares the received power values and adapting the data rate to an appropriate level ( $\max$ ), example $\mathrm{Rmax}=36 \mathrm{Mbps}$. At this stage, the data rate which a result from the $\mathrm{CF}$ algorithm is still less than the maximum level. Thus the new data rate for fading will stay at the same data rate as the ideal channel condition. As the load increase the cost function algorithm adapting the data rate to much higher level which is more than the limit ceiling rate. In fading condition, the data rate will be limit to this ceiling rate only. Therefore overall data rate for fading show lower values than the ideal case as the load increase. From this explanation, it can be concluded that, the energy saving for fading condition can be seen at much higher load due to adapting towards lower data rates for fading condition. 


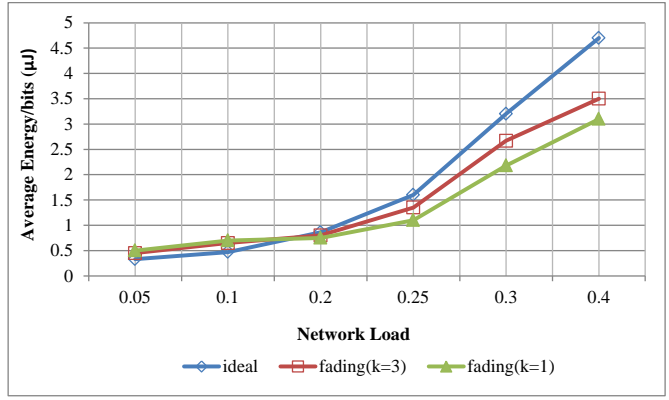

Figure 7. Comparison of energy per bits for all conditions with increasing load

Figure 8 shows comparison average of ete delay for all cases with increasing load. It can be seen from the figure that adaptation cost function in ideal channel results in better delay performance compares to combined cost function and adaptive rate/power in fading channel because adaptive rate in fading adapts to much lower transmission data rate than the ideal adaptation cost function thus contributes to more packet loss and higher retransmission, yet it gain advantage of major energy saving compare to other conditions, because that is the main goal of the proposed technique. However, due to high impaired channel especially at high load condition, the ete delay starts to increase at $(p=0.4)$ for fading case. As mentioned, since the proposed algorithm is not a safety critical application, thus slight increase of delay is acceptable at the cost of improved energy saving.

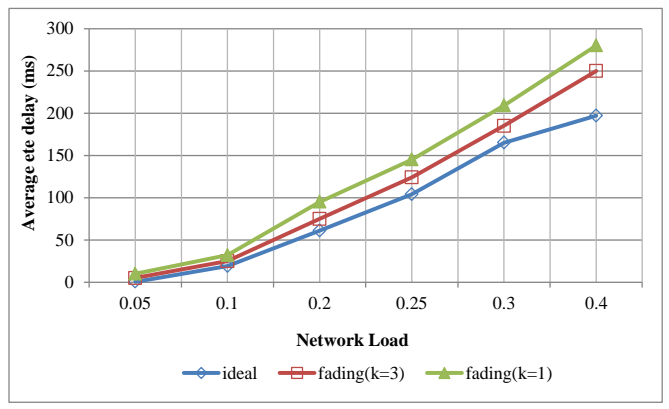

Figure 8. Comparison of average ete delay for all conditions with increasing

\section{CONCLUSION}

In ideal channel condition, the adaptation based on the cost function adapts the data rate according to the load conditions, which results in the changing of the data rates from lower rates (during low load conditions) and gradually moves to higher rates (at higher load conditions). However, in the fading channel, due to the varying nature of environment, a possible solution to combat the channel fluctuations is transmitting with more robust transmissions. It can be said that in a fading channel, the transmission data rate is independent of the load but depends solely on the fluctuations of the channel conditions which is represented by the received power value. Therefore, the enhance dual parameter cost function algorithm for fading makes use of the adaptation cost function with an additional feature to cope with the fading conditions by limiting the data rate according to the received power values.

\section{REFERENCES}

[1] C. Eyupoglu and M. A. Aydin, "Energy Efficiency in Backbone Networks," Procedia - Social and Behavioral Sciences, vol. 195, pp. 1966-1970, 7/3/ 2015.

[2] M. Meo, E. Le Rouzic, R. Cuevas, and C. Guerrero, "Research challenges on energy-efficient networking design," Computer Communications, vol. 50, pp. 187-195, 9/1/ 2014.

[3] "Cisco visual networking index: Forecast and methodology, 2011-2016," 2012.

[4] Hwi Young Lee, Won Jin Lee, "Channel allocation and transmission power management scheme in software defined network-based WLAN environments", 2017 International Conference on Information Networking (ICOIN), January 2017. 
[5] "Impact of IEEE 802.11n/ac PHY/MAC High Throughput Enhancements over Transport/Application Layer Protocols - A Survey", IEEE Communications Survey and Tutorials, February 2017.

[6] "IEEE 802.11ac Link Adaptation under Mobility", 2017 IEEE 42nd Conference on Local Computer Networks, Raja Karmakar, Samiran Chattopadhyay, 2017

[7] Thomas Chapman, Erik Larsson, Peter von Wrycza, Erik Dahlman, Stefan Parkvall, Johan Sköld, "Chapter 5 Scheduling, link adaptation, and hybrid-ARQ”, Editor(s): Thomas Chapman, Erik Larsson, Peter von Wrycza, Erik Dahlman, Stefan Parkvall, Johan Sköld, HSPA Evolution, Academic Press, 2015, Pages 71-87,

[8] M. Á Vázquez, M. R. B. Shankar, C. Kourogiorgas," Precoding, Scheduling and Link Adaptation in Mobile Interactive Multibeam Satellite Systems", IEEE Journal on Selected Areas in Communications, 2018

[9] Inaki Ucar, Carlos Donato, Pablo Serrano, Andres Garcia-Saavedra, Arturo Azcorra, Albert Banchs, "On the energy efficiency of rate and transmission power control in 802.11",Computer Communications, Volume 117, 2018, Pages 164-174,

[10] Juan J. Gálvez, Pedro M. Ruiz, "Joint link rate allocation, routing and channel assignment in multi-rate multichannel wireless networks", Ad Hoc Networks, Volume 29, 2015, Pages 78-98,

[11] Q. Dong, K. Hayashi, M. Kaneko, "A New Adaptive Modulation and Coding Method for Communication-Based Train Control Systems using WLAN”, IFAC-Papers On Line, Volume 49, Issue 22, 2016, Pages 139-144,

[12] Tingpei Huang, Shibao Li, Shaoshu Gao, "RaCA: A joint rate and channel adaptation scheme for dense $802.11 \mathrm{n}$ networks", Procedia Computer Science, Volume 111, 2017, Pages 183-189,

[13] J. Wang, X. Zhang, "Heterogeneous QoS-Driven Resource Adaptation over Full-Duplex Relay Networks", 2016 IEEE Global Communications Conference (GLOBECOM), 2016.

[14] L. Wang, X. Qi, J. Xiao, "Exploring Smart Pilot for Wireless Rate Adaptation", IEEE Transactions on Wireless Communications. Vol 15, Issue 7, 1536-1276, 2016

[15] Raja Karmakar, Samiran Chattopadhyay, Sandip Chakraborty, "SmartLA: Reinforcement learning-based link adaptation for high throughput wireless access networks", Computer Communications, Volume 110, 2017, Pages 125 ,

[16] Ke Wang, Teck Yoong Chai, Wai-Choong Wong, "Routing, power control and rate adaptation: A Q-learning-based cross-layer design", Computer Networks, Volume 102, 2016, Pages 20-37,

[17] Wensheng Lin, Bo Li, Mao Yang, "Integrated Link-System Level Simulation Platform for the Next Generation WLAN - IEEE 802.11ax", 2016 IEEE Global Communications Conference (GLOBECOM), 2016.

[18] W. N. W. Muhamad,J. Y. Khan, J. Brown, "Performance analysis of a tunable cost function based WLAN energy efficient packet transmission technique”, 2014 IEEE Asia Pacific Conference on Wireless and Mobile, 2014. 